\title{
UN JANUS À DEUX VISAGES. PATRIMONIALISATIONS DU RELIGIEUX INITIATIQUE ET DISCOURS DE LA TRADITION DANS LES MUSIQUES «TRADI-MODERNES » DU GABON Alice Aterianus-Owanga
}

Presses de Sciences Po (P.F.N.S.P.) | « Autrepart »

2016/2 N 78-79 | pages 103 à 124

ISSN 1278-3986

ISBN 9782724634402

Article disponible en ligne à l'adresse :

http://www.cairn.info/revue-autrepart-2016-2-page-103.htm

\section{Pour citer cet article :}

Alice Aterianus-Owanga, « Un Janus à deux visages. Patrimonialisations du religieux initiatique et discours de la tradition dans les musiques «tradi-modernes » du Gabon », Autrepart 2016/2 (N 78-79), p. 103-124.

DOI 10.3917/autr.078.0103

Distribution électronique Cairn.info pour Presses de Sciences Po (P.F.N.S.P.).

(C) Presses de Sciences Po (P.F.N.S.P.). Tous droits réservés pour tous pays.

La reproduction ou représentation de cet article, notamment par photocopie, n'est autorisée que dans les limites des conditions générales d'utilisation du site ou, le cas échéant, des conditions générales de la licence souscrite par votre établissement. Toute autre reproduction ou représentation, en tout ou partie, sous quelque forme et de quelque manière que ce soit, est interdite sauf accord préalable et écrit de l'éditeur, en dehors des cas prévus par la législation en vigueur en France. Il est précisé que son stockage dans une base de données est également interdit. 


\title{
Un Janus à deux visages. Patrimonialisations du religieux initiatique et discours de la tradition dans les musiques " tradi-modernes " du Gabon
}

\author{
Alice Aterianus-Owanga*
}

Depuis quelques années, des études sur les musiques donnent la part belle à la notion de patrimoine, selon des perspectives diverses : alors que la notion de « patrimoine musical » était autrefois employée dans une tendance essentialisante, les travaux contemporains s'attachent davantage à examiner les lieux, les acteurs et les institutions impliqués dans le processus de patrimonialisation, et à décrire un patrimoine en train de se faire [Desroches et al. 2011 ; Olivier ; 2011 ; CharlesDominique 2013]. Récemment, plusieurs publications se sont penchées sur des genres musicaux ou sur des pratiques ayant été reconnus comme patrimoine mondial par 1'Unesco [Samson, Sandroni, 2013], quelques autres sur des festivals [Campos, 2011], des groupes de musique, des politiques culturelles [Pourrouquet, 2015] ou des associations [Roda, 2011] participant de la patrimonialisation des musiques.

Prolongeant ce champ de recherche sur les liens entre patrimoine et musique, le présent article s'attache lui aussi à dépasser les approches opposant patrimonialisations par le haut et par le bas pour penser des patrimonialisations « ordinaires » [Isnart, 2012]. Il ne s'intéresse ainsi pas à la patrimonialisation institutionnelle d'une musique, mais à la manière dont des musiciens contribuent à la mise en patrimoine de certaines pratiques culturelles, et aux mobilités qui conduisent à faire émerger chez ces artistes un projet d'ordre patrimonial de valorisation de traits culturels ou d'objets perçus comme « traditionnels ». L'hypothèse sous-jacente à cette démarche repose sur l'idée que l'anthropologie du transnational et de la mondialisation appliquée aux trajectoires musiciennes [White, 2012 ; Le Menestrel, 2012] permet d'éclairer des manières particulières de produire du « patrimoine », de la «tradition » et des «identités » par la mobilité, ainsi que des étapes, des logiques et des tensions qui jalonnent ce processus.

\footnotetext{
* Anthropologue, maître-assistante FNS Ambizione, université de Lausanne.
} 
La réflexion ici présentée est fondée sur une ethnographie des mondes de la musique urbaine du Gabon ${ }^{1}$, un cas d'étude qui offre des particularités remarquables dans les manières de faire patrimoine. En effet, l'État gabonais ne s'est engagé que très relativement et tardivement en faveur d'une politique d' "authenticité » ou d' « enracinement culturel » [White, 2006 ; Ndiouga Benga, 2008], et a appréhendé avec méfiance les démarches qui s'écartaient du projet d'unité nationale du Parti unique. Le Gabon n'est pas célébré pour ses patrimoines musicaux ${ }^{2}$ : aucun de ses chants, danses, et pratiques cérémonielles n'est inscrit au patrimoine culturel immatériel de l'Unesco ni ne s'est érigé dans les marchés world-music comme emblème d'une sonorité nationale, reconnue depuis l'international comme le sont le mbalax pour le Sénégal, le highlife pour le Ghana, ou le samba de roda pour le Brésil. Hormis quelques exceptions, peu de ses artistes ont rencontré la consécration dans les secteurs de commercialisation des musiques africaines.

Pourtant, depuis les années 1950, c'est autour des musiciens que s'est en partie formée, exprimée et diffusée au Gabon l'idée d'une identité nationale « moderne », d'émotions et d'ethos communs, ainsi qu'un projet étatique d'unité nationale. Des années 1960 aux années 1990, plusieurs chanteurs se sont érigés en hérauts d'un projet de « retour aux sources », et ils se sont fait les initiateurs du passage à l'art [Heinich, Shapiro, 2012] d'éléments de traditions initiatiques, dont certaines s'imposèrent par la suite comme patrimoines nationaux. Ces artistes ont progressivement été érigés en représentants d'un label musical dit "tradi-moderne », selon une terminologie employée au Gabon et dans d'autres États africains [White, 2012, p. 205]. Cette étiquette musicale locale réunit des artistes de genres musicaux différents, mais partageant une même représentation des cultures postcoloniales contemporaines comme possibles produits d'une heureuse rencontre entre « tradition » et « modernité ».

En décrivant les trajectoires de trois musiciens engagés dans ce travail de mise en patrimoine de pratiques culturelles locales ${ }^{3}$, cet article examine les circulations locales et transnationales qui ont conduit à ce processus, ainsi que les déplacements d'objets ou de pratiques qu'il a entraînés. Il s'organise en suivant les trois types de mobilités et de mécanismes de patrimonialisation sous-jacents dans ces parcours :

1. Cette réflexion est le fruit des recherches menées à Libreville de 2008 à 2015, financées grâce à une allocation doctorale de l'université Lyon 2, puis des allocations postdoctorales de l'APRAS et du Labex CAP.

2. Un seul bien est inscrit sur la liste du patrimoine mondial de l'Unesco : l' « écosystème et paysage culturel relique de Lopé-Okanda ».

3. D'autres artistes se sont également impliqués dans une démarche proche, comme Pierre-Claver Zeng [Mvé Békalé, 2000; Ondo, Ovono Ebé, 2014]. Les trois artistes ici décrits ont été choisis en raison de l'existence de nombreuses archives à leur propos, de la possibilité d'effectuer une investigation ethnographique dense sur leur parcours et des éclairages qu'ils fournissent à propos des mécanismes de la patrimonialisation au Gabon. 
- la première mobilité se déroule à partir des années 1960 sur les routes du panafricanisme, et conduit à une appropriation de traits culturels perçus comme authentiquement africains, pour lutter contre l'aliénation coloniale ;

- la seconde repose, à partir des années 1970, sur des mobilités artistiques dans des scènes nationales et internationales de musique africaine, qui se prolongeront dans les marchés étiquetés plus tard world-music; elle s'associe avec une logique de patrimonialisation ambiguë, consistant en la spectacularisation d'éléments religieux et la désactivation de leurs significations symboliques, afin d'en faire des signes identitaires;

- la dernière est une mobilité d'objets, de symboles et d'images consécutive aux processus de spectacularisation de pratiques initiatiques, observée à partir des années 1980 : elle entraîne la décontextualisation et la resignification des éléments patrimonialisés.

En décrivant ces mobilités, nous examinerons la contribution méconnue du musical au passage d'une pratique religieuse initiatique au patrimoine, en prolongement de travaux qui ont souligné la manière dont le bwiti s'était érigé en emblème d'un patrimoine national [Mary, 2005 ; Bonhomme, 2007]. Au préalable, une première partie de cet article resitue le contexte historique et politique de déploiement de ces pratiques musicales et des politiques culturelles entourant l'indépendance.

\section{Des soubassements politiques du patrimoine : nations et nationalismes au Gabon}

Les travaux sur les enjeux, usages et contours de la notion de patrimoine ont de longue date relevée comment cette catégorie fonctionnait en interdépendance avec celles « d'identité », de « tradition » ou de « culture » [Fabre, 1994 ; Le Goff, 1998]. Tandis que les approches historiques ont montré comment le cadre de la nation et de l'État modernes avait constitué une matrice de formation de cette idée de patrimoine dans le contexte européen [Pomian, 2010], des approches anthropologiques plus récentes ont examiné les enjeux politiques de sa diffusion et de sa localisation dans des États variés [Bondaz et al., 2014 ; Givre, Regnault, 2015], soulevant la question des rapports entre nationalisme, État et patrimoine.

En Afrique, l'entrecroisement entre dynamiques de patrimonialisation et constructions nationales fut particulièrement remarquable au tournant des indépendances. Durant cette période, les nouvelles nations s'attachaient à produire des identifications libérées de l'aliénation coloniale, associant le projet développementaliste à la démarche d'affirmation de «traditions » ou de «patrimoines ». Le Gabon offre un exemple intéressant de la manière dont cette opération survint par un processus d'interactions et de négociations incluant des acteurs situés à la fois au sein et en dehors du cadre étatique, à l'échelle locale et globale. 
Le Gabon colonial n'est pas connu pour ses mouvements indépendantistes et ses revendications anticoloniales. Traversés par des conflits de classe et des luttes de pouvoir [Bernault, 1996], les partis politiques se positionnent, par exemple, en majorité en la défaveur de l'indépendance lors du référendum du 28 septembre 1958, percevant l'inclusion dans la communauté française comme plus favorable aux intérêts du Gabon que l'indépendance ou l'alliance au sein de regroupements sous-régionaux [M'Bokolo, 2009]. Ces partis politiques militent cependant tous pour l'affirmation d'une unité territoriale et revendiquent les intérêts de la nation gabonaise. Parallèlement, durant la première moitié du XX $\mathrm{XX}^{\mathrm{e}}$ siècle, des mouvements syndicaux, des groupes d'intellectuels et des associations d'étudiants se mobilisent de diverses manières, sinon pour l'indépendance, du moins pour l'amélioration des conditions de vie et de travail des Gabonais [Métégué N'Nah, 2006, p. 153 ; Rich, 2012]. Du côté religieux également, des mouvements millénaristes et réformistes émergent dans l'ethnie fang, annonçant la libération prochaine du peuple noir, et la venue d'un messie identifié en la personne de Léon M'Ba, futur premier président du Gabon [Mary, 1983a]. Quoique divergentes, ces initiatives révèlent la présence déjà marquée d'une idée de nation et de destin communs chez les peuples gabonais. Sous la pression de l'opinion publique et du vent de libération qui traverse le continent, l'indépendance du Gabon est finalement proclamée par Léon M'Ba le 17 août 1960.

Alors que le Gabon traverse cette période de transition, une scène musicale se développe dans Libreville, autour des sonorités circulant sur les voies de l'empire colonial. Au sein des débits de boisson et des bars-dancings, les citadins réunis par l'exode rural et les migrations adoptent les esthétiques, les danses et les pratiques de fêtes synonymes de «modernité ». Ces lieux accueillent progressivement les performances des orchestres naissant dans la ville. Ces orchestres bénéficient de l'aide de personnalités politiques et d'élites qui participent à l'acquisition d'instruments ou fournissent des lieux de répétition. Du fait de cette influence politique, des identifications ethniques ou provinciales sont assignées progressivement à ces formations musicales, chacune devenant l'ambassadrice d'un groupe ethnique ou d'une province gabonaise.

À partir de l'indépendance, cette proximité entre le domaine politique et le monde musical prend une nouvelle ampleur. Dès 1960, les musiciens d'orchestre sont sollicités pour élaborer l'hymne national, avant d'être impliqués dans l'animation des campagnes électorales. Comme le décrit Florence Bernault, bars et musiques forment alors les véhicules principaux d'une «culture quotidienne du politique » en développement [Bernault, 1996, p. 311], orchestrant le «ballet complexe du dialogue entre gouvernement et population » [Ibid, p. 323]. La présidence de Léon M'Ba, de 1960 à 1967, voit aussi le développement de plusieurs institutions étatiques, en adéquation avec les «accords de coopération » signés avec la France, dont l'influence se remarquera dans différents secteurs [Obiang, 2007]. Dans le domaine de la culture, le Musée national des arts et traditions voit le jour, ainsi que la Direction de la culture et les antennes de communications télévisuelles et radiophoniques nationales, où seront enregistrées les premières 
productions musicales locales. Cependant, les troubles politiques et le climat autoritaire sous le régime de Léon M'Ba freinent le développement des politiques culturelles, et les musiciens sont eux-mêmes touchés par la répression, l'instabilité et la fragilité de l'État postcolonial.

C'est véritablement avec l'arrivée au pouvoir d'Omar Bongo que se développe l'appareil d'organisation et de contrôle du monde de la culture. Vice-président de Léon M'Ba, Albert Bernard Bongo ${ }^{4}$ arrive au pouvoir en 1967, et abroge le multipartisme pour créer le 12 mars 1968 le Parti démocratique gabonais (PDG). Avec son idéologie de «Rénovation », il promeut le développement de la nation par l'unité des «tribus », des «clans » et des « ethnies» autour de la figure centrale du président. Après la période de division et d'ethnicisation de la vie politique sous Léon M'Ba, Omar Bongo entend faire régner l'unité nationale, se fondant pour ce faire «sur la négativation des cultures » [Nguéma-Minko, 2010, p. 171], et sur l'effacement des particularismes locaux. Il assoit son hégémonie sur une centralisation politique et institutionnelle, sur des services de sécurité et de renseignement, et sur des réseaux d'interdépendance avec l'ancienne métropole [Obiang, 2007], mais également sur la musique et la danse.

Dans le domaine musical, le PDG contrôle les contenus de la création au travers de deux types d'instruments d'enrôlement : d'une part, à partir de 1968, les forces armées nationales créent les orchestres militaires et réquisitionnent les musiciens des groupes existants, pour exercer leurs talents au service des institutions. Leurs performances répondent aux commandes des généraux et des dirigeants politiques, pour animer les festivités et cérémonies officielles. D'autre part, les «groupes d'animations socioculturels » se mettent en place sous la dépendance de l'UFDPG (l'Union des Femmes du PDG), réunissant les femmes par appartenances provinciales ou ethniques. Gérés par des personnalités politiques ou leurs épouses, ces groupes féminins animent les cérémonies officielles par des chants et par des danses insistant sur l'érotisme et les mouvements du bassin [Mouélé, 2009 ; Aterianus-Owanga, à paraître], et ils obéissent à une division genrée des activités culturelles sous le régime du PDG. En leur sein, les musiciens et danseurs sont aux prises avec des injonctions contradictoires : d'une part, ils sont contraints à la recherche d'une certaine « authenticité » africaine par les discours politiques, les critiques des médias et par les appels plus globaux à la réafricanisation culturelle sur le continent; d'autre part, leurs élans de recours à des langues et sonorités ethniques sont freinés, car perçus comme des menaces à l'unité nationale, et l'on privilégie la reprise des standards musicaux reconnus dans les marchés de la musique. Ces contradictions reflètent les ambivalences accompagnant le rapport à la «tradition» dans les premières heures du régime Bongo.

En contraste avec ce tableau de politisation extrême des musiciens et ces attitudes ambiguës à l'égard du registre de la «tradition », plusieurs artistes

4. Albert Bernard Bongo prend le nom El Hadj Omar Bongo en 1973 à la suite de sa conversion à la religion musulmane. 
parviennent à se positionner en marge ou dans les interstices du système politique, pour développer d'autres démarches d'invention de traditions musicales, religieuses et orales. Pour ce faire, leurs créations musicales s'inspirent de circulations et de dialogues en direction de l'étranger, dans les réseaux panafricanistes.

\section{Panafricanisme et décolonisation culturelle : la patrimonialisation en exil de Pierre-Claver Akendengué}

\section{Chanter l'Afrique, entre la France et le Gabon}

Dans les années 1960, après l'indépendance du Ghana en 1957, le continent africain voit le fleurissement des expressions du panafricanisme, un mouvement hétérogène, mais possédant pour socle commun le projet d'unité des peuples de l'Afrique et de sa diaspora [Adi, Sherwood, 2003; Boukari-Yabara, 2014]. Porté sur le continent par des personnalités politiques telles que Kwame Nkrumah, Jomo Kenyatta et Sékou Touré, le panafricanisme naît des dialogues avec les théoriciens du monde noir des Amériques, comme William Edward Burghardt Du Bois et Marcus Garvey. Au-delà du registre politique, les festivals culturels panafricains tenus entre 1966 et 1977 s'attellent à diffuser ce mouvement idéologique auprès du plus grand nombre, constituant des plateformes inédites de rencontre et de mise en réseau des artistes africains.

Le panafricanisme exerça un impact bien moindre et bien plus tardif en Afrique centrale que dans les zones occidentales et orientales du continent [M'Bokolo, 2009]. Au Gabon, sous Omar Bongo, bien qu'un panafricanisme « raisonné » se développe en vue d'alliances diplomatiques, économiques et d'influences géostratégiques [Ibid.], les initiatives émancipatrices se rattachant au panafricanisme restent mises sous silence, soupçonnées de menacer l'hégémonie du PDG. Pourtant, quelques artistes en mobilité vont s'en imprégner pour développer une entreprise de « retour aux sources » et de nationalisme culturel qui essaimera à diverses échelles.

Pierre-Claver Akendengué représente le plus célèbre orateur de ce discours d'unité africaine et d'émancipation culturelle au Gabon. Évoluant durant une longue période sur les scènes musicales étrangères, au départ dans les milieux des étudiants africains de France, puis en exil, il fut le premier artiste à faire connaître une «musique gabonaise » auprès des médias et publics étrangers. Pionnier du secteur marchand de la world-music, il fut aussi le premier à se saisir d'éléments vocaux, instrumentaux et rythmiques puisés dans les musiques religieuses initiatiques.

Né en 1943 à Awuta, dans la région des lacs du Gabon, Pierre-Claver Akendengué grandit entre Port-Gentil, capitale économique, et le contexte villageois où il retourne passer ses vacances ${ }^{5}$. Il y assiste aux cérémonies initiatiques

5. Pour des biographies et analyses de l'œuvre de Pierre-Claver Akendengué, voir Tindy-Poaty [2008], Retondah [2012] et Mbondobari [2004]. 
de l'elombo ${ }^{6}$ et du bwiti $^{7}$, auprès de son oncle initié, guérisseur et remarquable joueur de ngombi, une harpe que l'on retrouve dans une partie du sud-est du Gabon, et qui revêt des fonctions sacrées dans le rituel [Sallée, 1985]. Les sonorités auxquelles il se familiarise l'amènent à élaborer un jeu de guitare particulier, transposant sur cet instrument occidental des phrasés et techniques musicales des harpistes. Il s'en inspire aussi pour adjoindre des chants féminins aux chœurs de ses morceaux, ce qui deviendra une caractéristique de son style chansonnier. Puis il assiste durant ses années au collège Bessieux aux premières heures de formation des orchestres gabonais, avant de partir en 1964 étudier en France, où il intègre une chorale et participe en 1967-1968 à un télé-crochet nommé le «Petit Conservatoire de Mireille ». C'est en son sein qu'il est incité à puiser dans les langues vernaculaires et les traditions gabonaises, et qu'il remporte le troisième prix du concours avec sa chanson «Nkéré », inspirée du style musical des rites initiatiques féminins du ndjembè. Il explique ainsi les influences de Mireille sur son orientation musicale :

« C'est d'ailleurs Mireille qui m'a convaincu de m'intéresser davantage à ma culture ancestrale, et à la culture africaine en général. C'est ainsi que grâce à elle, les premières chansons que j'ai enregistrées ont été résolument des chansons africaines. »

À la différence d'autres artistes gabonais de son époque, Pierre-Claver Akendengué s'inscrit dès ses débuts dans un parcours transnational, marqué par des allers et retours entre la France et son pays natal, qui lui permettent de s'autonomiser du contrôle des musiciens, et de découvrir d'autres formats de reconnaissance musicale, passant par la représentation de sa «culture ancestrale ».

Les poésies engagées d'Akendengué se rapprochent souvent du conte, habitées de personnages mythiques et d'animaux anthropomorphes. Regorgeant de railleries sur les vilénies de la classe politique, elles appellent à la libération de l'homme noir, gabonais et africain. Dans les chants d'Akendengué, ces personnages imaginaires évoluent aux côtés des figures de la résistance politique africaine, et ils s'insèrent au sein d'un discours panafricaniste plus global. Mbombi, Behanzin, Lumumba, Cabral, Kwame Nkrumah, Steve Biko et Malcolm X apparaissent comme autant d'enfants d'une mère patrie dont les limites ne se cloisonnent ni aux portes de la nation gabonaise ni à celles du continent africain, mais à celles qui englobent l'ensemble de l'Atlantique noir [Gilroy, 2010]. Comme lorsqu'il chante «tout nègre a le même combat, tout nègre a le même sang » dans le morceau « Négro » [Akendengué, 1976], il élève un chant d'unité de l'homme

6. L'elombo est une société thérapeutique mixte, créée dans les années 1930 par les Nkomi de la région côtière du Fernan-Vaz, qui se différencie du bwiti en ce qu'elle se fonde sur une expérience de possession, et non de vision [Mary, 1983a, p. 284].

7. Le bwiti (ou bwete) représente le plus célèbre et le plus répandu des rites initiatiques gabonais. Quoiqu'il possède des variantes nombreuses, une majorité de rites apparentés au bwiti se caractérisent par une initiation fondée sur la consommation d'un hallucinogène local, l'iboga, vecteur d'un voyage visionnaire. Une dynamique de secret régit la circulation du savoir au sein de cette société d'initiés, et les logiques d'aînesse initiatique déterminent son organisation hiérarchique [Bonhomme, 2006]. 
noir. Germée dans le creuset de la diaspora africaine de France, l'invocation de libération du sujet noir de Pierre-Claver Akendengué s'inscrit de la sorte dans la continuité des projets identitaires du nationalisme culturel africain qu'il rencontre dans ses mobilités.

Soupçonné de militer contre le PDG au sein de l'association générale des étudiants gabonais (AGEG), considéré comme un réseau d'opposition au parti unique [Retondah, 2012], Akendengué est interdit d'antenne et de séjour au Gabon à partir de 1972, ce qui le contraint à vivre en exil en France jusqu'en 1985. Quoique résident à l'étranger, il s'impose durant vingt années sur la scène internationale et au Gabon comme figure emblématique de la nation gabonaise et comme chantre de la «chanson engagée ». Dans les années 1970 et 1980, les relations qu'il possède auprès de quelques personnalités puissantes l'aident à enregistrer des albums destinés au Gabon et exercent des pressions contre son interdiction de diffusion. Ces soutiens et la montée en puissance de sa carrière dans les réseaux internationaux de musique du monde permettront finalement sa réhabilitation. En 1985, il décide de mettre fin à son exil, lassé de l'éloignement des situations sociales et politiques qu'il s'attache à dénoncer dans ses morceaux. De retour à Libreville, il met en place des structures de promotion des jeunes musiciens, et il accepte l'invitation à occuper des fonctions de conseiller à la présidence, tout en conservant un discours très critique à l'égard des réalités politiques locales.

\section{"L'Afrique spirituelle » : contre-discours et icône marchande}

Outre ses connexions à la pensée panafricaine, la France est aussi pour Akendengué le lieu de rencontre avec les producteurs inscrits dans les démarches embryonnaires de promotion des musiques «autres». Comme Mireille et plus tard Pierre Barouh, qui produit ses premiers disques dans la société Saravah, ces acteurs de la nouvelle scène de chanson française influenceront la construction de son style tourné vers les «traditions». Il se met également en contact avec des musiciens brésiliens, haïtiens ou antillais, auprès de qui il développe une réflexion sur l'organologie «négro-africaine » et les musiques «d'inspiration africaine » [Arnaud, Akendengué, 2008]. Enfin, il trouve un appui puissant auprès des mouvements militants de gauche qui l'invitent dans des meetings à «représenter l'Afrique » [Ibid.].

Pierre-Claver Akendengué expérimente ainsi en France des collaborations qui le mettent en première ligne des marchés naissants de la world-music. Confronté à ces structures de production, il tâche de négocier et de créer un compromis entre son projet panafricain d'émancipation culturelle et l'accès à des réseaux de diffusion porteurs de regards stéréotypés sur l'Afrique. Il énonce ainsi sa critique des clichés sur l'africanité imprégnant ces marchés et sa proposition de représentations alternatives de l'Afrique : 
«L'Afrique a souffert de l'éclipse identitaire. On nous a réduits à un décor sauvage, folklorique, auquel il faut ajouter le système économico-militaire qui nous a gérés. Tout ça a donné de l'Afrique une image abâtardie. Avec comme postulat : l'Afrique, c'est la danse ou des trucs de ce genre. Alors que chez nous existent des forces spirituelles. Il y a l'Afrique animiste, l'Afrique profondément religieuse. Je crois qu'il nous appartient de maîtriser cette spiritualité et, dans le mouvement du donner et de recevoir, de la proposer aux autres. » (Akendengué interviewé par Thiéfaine, 1996, p. 134)

Comme en témoigne le cliché de l'Afrique dansante à laquelle il s'oppose, les marchés de la world-music auxquels Pierre-Claver Akendengué se confronte s'avèrent marqués de l'empreinte de l'« exotique postcolonial » [Huggan, 2001 ; White, 2012]. Chez Pierre-Claver Akendengué, la philosophie de libération et d'autodéfinition du soi africain requiert de s'émanciper de ces visions folkloristes et exotiques, pour proposer d'autres images de l'africanité, en l'occurrence ici des images de spiritualité. Cette démarche, imprégnée d'une représentation de la mondialisation comme heureux dialogue des cultures, découle en partie de paradigmes qui nourriront les rencontres et les productions étiquetées sous le label world-music [Feld, 2004].

Simultanément, il est difficile de comprendre cette part prégnante du religieux dans le projet culturel de Pierre-Claver Akendengué sans prendre en compte l'influence de l'anthropologie africaniste sur son discours. En effet, après avoir suivi des cours d'ethnomusicologie au Musée des arts et traditions populaires dans les années 1970, il soutient en 1986 une thèse de doctorat sous la direction de Louis-Vincent Thomas, intitulée «Religion et éducation traditionnelle en pays Nkomi au XIX siècle » [Akendengué, 1986]. Il se familiarise par ce biais avec une ethnologique africaniste qui demeure encore fortement imprégnée à l'époque du projet de connaissance des « sociétés traditionnelles », et de valorisation des peuples africains par la description de leurs spiritualités et principes ésotériques. Dans son projet de transmission de savoirs ancestraux «traditionnels » par la musique, il reprend en partie à son compte ce « vaste palimpseste [africaniste] qui se laisse assez bien synthétiser par la formule "tradition/modernité" » [Dozon, 2015, p. 67], et il se positionne, comme d'autres passeurs de mémoire, en «miroir du discours ethnologique » [Ciarcia, 2012, p. 9]. Tout en s'attelant à critiquer l'existence de stéréotypes sur l'africanité, il bâtit de fait sa reconnaissance internationale en rejouant d'autres stéréotypes sur l'africanité et les sociétés « traditionnelles », ceux de l'Afrique profonde, mystérieuse et animiste, en partie nourris de l'imaginaire africaniste.

Le rôle précurseur de Pierre-Claver Akendengué pour la mise en musique d'éléments traditionnels et leur cristallisation autour du religieux résulte, comme on le voit ici, de différents facteurs. D'une part, la mobilité translocale et les souvenirs de l'origine villageoise constituent la matrice d'une nostalgie et d'émotions au fondement de son projet patrimonial. Par ailleurs, l'engagement politique et idéologique induit par sa mobilité sur les routes du panafricanisme, puis sa rencontre avec les entrepreneurs de la musique en France alimentent sa démarche 
de recours à des éléments musicaux, philosophiques et religieux « traditionnels ». Enfin, les paradigmes qu'il mobilise s'ancrent dans des schémas et des visions de la «tradition» encore fortement imprégnés de l'africanisme ethnologique. Sa consécration est finalement rendue possible par des négociations avec le pouvoir et des compromis avec les différents régimes de conception de la «tradition », entre le régime de rénovation d'Omar Bongo, le nationalisme culturel panafricaniste, et les marchés musicaux de l'exotique postcolonial.

Toute une génération d'artistes gabonais poursuivra cette entreprise d'utilisation de la musique comme véhicule d'une langue, de savoirs et de pratiques locales, et comme socle de mélanges avec les sonorités «modernes » adoptées des flux globaux.

\section{Mobilités sous contrôle : la patrimonialisation ambiguë d'Aziz'Inanga}

\section{Parrainages genrés et négociations politiques}

Dans le sillage de Pierre-Claver Akendengué, plusieurs musiciens s'engagent cette démarche de mise en scène d'éléments traditionnels - costumes, danses, instruments et maquillages -, en explorant d'autres facettes de ces mêmes marchés de la musique africaine (foires internationales, festivals, studios d'enregistrement en France). La chanteuse Aziz'Inanga donne à voir l'une des trajectoires les plus intéressantes à ce propos et elle permet d'aborder une autre modalité de construction de patrimoines en mobilité ${ }^{8}$. Aujourd'hui retirée de la scène musicale, Aziz'Inanga a représenté pendant vingt ans une figure centrale de la scène gabonaise.

Née en 1951 dans la région des lacs du centre du Gabon, Aziz'Inanga est élevée auprès d'un père adepte de rhythm'n'blues et de chants traditionnels, puis dans un collège de sœurs catholiques. Influencée par la chanson religieuse, elle commence très jeune à exécuter des prestations vocales pour des festivités familiales ou religieuses (baptêmes, mariages, communions). Arrivée à Libreville après des études supérieures à Douala au début des années 1970, elle intègre l'ORSTOM où elle travaille comme statisticienne, et elle entre dans une troupe de théâtre dirigée par celui qui deviendra son concubin, manager et le père de ses enfants, François Rosira Nkiélo. Il lance sa carrière, et, grâce à ses connaissances dans les réseaux politiques et maçonniques gabonais, obtient les mécénats requis pour la production. Dès ses débuts, avec le «Théâtre du silence », elle effectue des voyages dans des foires internationales, comme en 1972, à Casablanca et aux États-Unis. Alors que le Gabon ne bénéficie pas encore de studio d'enregistrement ou de presse de disques microsillons, Aziz'Inanga se rend à plusieurs reprises en France, où elle entre en contact avec les milieux de la musique africaine et

8. Je m'appuie pour la description proposée sur des entretiens avec la chanteuse, ses proches, d'autres musiciens, et sur une analyse de la presse de l'époque. Ce travail est aussi lié à un film documentaire sur le parcours de vie d'Aziz'Inanga, en cours de finition au moment de l'écriture de cet article. 
caribéenne. Elle représente aussi le Gabon en 1977 au FESTAC de Lagos. À son retour de ce festival panafricain, un sondage la présente comme l'une des personnalités favorites des Gabonais [Mapangou, Barbier Decrozes, 1985]. Ainsi, avant même de connaître la consécration au Gabon, c'est dans les voies de diffusion internationales qu'elle rencontre la reconnaissance ${ }^{9}$. Les journaux locaux l'érigent en ambassadrice de la nation et relatent les épisodes de chacun de ses déplacements en dehors du Gabon.

Durant sa carrière, Aziz'Inanga est fréquemment sollicitée par le président Omar Bongo ou par des ambassadeurs du Gabon pour représenter son pays lors de festivités nationales. Ses mobilités s'exercent ainsi en partie dans des réseaux encore fortement rattachés aux autorités politiques, même si elle s'en écarte aussi pour tisser ses propres systèmes de production, et refuse l'inclusion au sein des « groupes d'animation » où sont confinées de nombreuses femmes chanteuses de l'époque. Pour Aziz'Inanga, les possibilités de développement de carrière sont alors largement déterminées par une organisation genrée du monde du spectacle et de la culture ${ }^{10}$. C'est grâce aux réseaux d'interconnaissance de son conjoint qu'elle parvient à négocier cette relative autonomie vis-à-vis du système Bongo, et à faire par exemple financer son dernier album par l'ACCT, qui l'amènera à se produire en Louisiane et au Canada. En enregistrant des morceaux en langue myènè, punu et fang, Aziz'Inanga suit les injonctions d'unité nationale énoncées par l'État et elle affirme une volonté de dépassement des clivages ethniques, pour chanter la nation gabonaise. Parallèlement, c'est en usant du double langage et des sous-entendus contenus dans ses chants en langue myènè qu'elle transmet une perspective critique sur le régime Bongo, le discours de la tradition constituant alors le creuset de la subversion.

Surnommée souvent dans la presse la femme-panthère, animal emblème du Gabon, Aziz'Inanga en vient, comme d'autres artistes de son époque, à incarner un agent du patrimoine, en ce qu'elle opère le passage ou la transmission (réinvention) d'éléments ou de pratiques issus du passé.

\section{Conversions religieuses et paradoxes de la « tradition » initiatique au Gabon}

Ainsi investie d'une responsabilité patrimoniale, Aziz'Inanga se fait plus particulièrement connaître comme l'une des artistes puisant dans les danses et chants féminins typiques des sociétés initiatiques gabonaises pour créer son style « tradimoderne ». Dotée d'une voix aux modulations complexes et déconcertantes, variant des sonorités les plus aigües aux plus graves, Aziz'Inanga s'attelle dans ses morceaux à se faire l'énonciatrice de valeurs, de conseils et de préceptes ancrés

9. Dans les scènes et festivals internationaux, les médias apprécient alors les formes enchanteresses de la jeune chanteuse, sa « félinité » et ses mélodies rythmées, y voyant un chantre de « l'Afrique profonde, l'Afrique mystérieuse, la vraie Afrique », selon des discours aux accents souvent primitivistes et stéréotypés.

10. Bien qu'elle soit toujours d'actualité dans les mondes de la musique urbaine [Aterianus-Owanga, sous presse], cette division genrée n'est pas le propre du Gabon. Voir à ce propos Buscatto [2007]. 
dans l'univers villageois de son enfance. Ainsi dans son premier album, elle narre la nostalgie des pirogues à voile («akuku», 1979), disparues au profit des bateaux à moteur, à l'image de tant de pratiques mises de côté par l'urbanisation. Au fil de sa carrière et de ces mobilités, son style musical évolue, du discours de retour aux sources et de valorisation des cultures traditionnelles à des ambiances jazz, blues ou rumba, pour tendre vers un style «world», fusionnant les sonorités des synthétiseurs avec ses chants en langues gabonaises.

Dès ses premiers albums, les instruments occidentaux qui accompagnent ses chants associent des éléments rythmiques caractéristiques du ndjembè (société initiatique féminine), de l'élombo et du bwiti, tandis que sur scène, les ceintures de raphias qu'elle porte aux hanches ou sur la tête et les mouvements de bassins qu'elle exécute s'inspirent très clairement des tenues et danses d'initiées, de même que certains maquillages qu'elle arbore. La tenue d'Aziz'Inanga est alors l'un des motifs principaux du rattachement qui est opéré localement entre cette artiste et les sociétés initiatiques locales. En effet, dans ces rituels, le voyage visionnaire s'accompagne souvent de l'acquisition d'un nom d'initié, d'une connexion avec un esprit tutélaire, et d'une identification à des animaux ou entités totémiques, manifestés par le port de peaux d'animaux et par des maquillages particuliers [Bonhomme, 2006]. La peau de genette portée par Aziz'Inanga et d'autres chanteurs de cette époque est issue de ces costumes arborés par les initiés [AterianusOwanga, 2015].

Pour beaucoup d'auditeurs et de pairs du milieu de la musique, l'appartenance d'Aziz'Inanga à la société des initiés ne fait donc aucun doute, et elle fonderait sa démarche d'utilisation d'éléments issus du champ religieux sur les scènes musicales. Pourtant, la chanteuse refuse cette identification au domaine initiatique, et elle l'associe avant tout à l'héritage culturel légué par son père, lui-même initié. Elle s'attache à affirmer sa distance à l'égard de ce registre traditionnel avec d'autant plus d'insistance qu'elle devient «sœur en christ» en 1995. Elle se convertit à l'époque au pentecôtisme (au sein de l'Église du Mont Sinaï), en vue de trouver la résolution à des soucis de santé, des impasses économiques et des difficultés dans sa carrière. Au moment de nos échanges, elle explique ainsi l'origine de sa tenue :

«Je portais des peaux de bête. J'expliquais aux gens pourquoi : tout simplement pour faire connaître aux gens que nous n'avons pas toujours été ce que nous sommes, et dans la Bible, le premier vêtement que Dieu a fait porter à l'homme, c'était des peaux de bête. Ça n'a rien à voir avec une initiation quelconque, je ne suis pas initiée à quoi que ce soit dans les rites traditionnels. Je connais pas. Mais les gens ont tendance à penser que quand tu te mets une tenue traditionnelle, c'est comme si tu te mettais dans les initiations. [...] Moi, c'était symbolique, j'aimais bien ma tenue ; je l'aime, je l'ai créée moi-même, elle n'a pas été inspirée. C'était pour revendiquer le côté africain, on n'a pas honte de ce qu'on est. » (Aziz'Inanga, mai 2012, Libreville) 
Dans l'explication ci-dessus, c'est toujours la revendication d'un retour aux sources africaines qui justifie les mises en scène de l'initiatique dans le spectacle de l'africanité de la chanteuse. Cependant à la différence de Pierre-Claver Akendengué, qui décrivait cet univers avec nostalgie et admiration, l'utilisation d'éléments initiatiques s'associe chez Aziz'Inanga à une évacuation de leur référent religieux, pour en faire des constituants d'un environnement culturel plus global, selon un processus de «métaphorisation du religieux » [Hervieu-Léger, 1993]. Le recours au symbole initiatique est combiné avec un rejet du registre symbolique dont il est extrait. Au contraire, le discours de retour aux origines africaines se «branche» [Amselle, 2001] avec un message chrétien, ce qui résulte des influences des missions évangélisatrices et de l'incorporation profonde de la chrétienté au Gabon, mais surtout des reconfigurations induites par la conversion de la chanteuse au pentecôtisme.

De la sorte, l'exemple d'Aziz'Inanga met en évidence les lignes de tension résultant des mises en mouvement d'objets et de symboles religieux en dehors de leurs contextes initiaux. Steven Feld aborde ce processus en matière de «recontextualisation » et de «resignification» des sons en dehors de leurs contextes d'origine, en soulignant le pouvoir de représentation de l'authenticité conféré au domaine religieux ou spirituel dans les marchés de la musique [Feld, 2012].

Le processus de cristallisation des cultures «autres » autour du spirituel est exacerbé dans les œuvres et la réception des musiciens de cette période : les discours de retour aux sources africaines entretenus par certains artistes, puis l'ère d'expansion de la world-music dans les marchés de l'art (à partir des années 1980) et sa production de la valeur autour d'éléments traditionnels revisités investissent ces objets d'une nouvelle valeur, une valeur patrimoniale. En outre, le passage à la scène musicale conduit lui-même à une transformation de ces matériaux religieux, mués en icônes spectacularisées: les maquillages et parures initiatiques deviennent des métaphores de la tradition et de la «gabonité » invoquée, leur sens religieux se voyant désactivé.

Comme pour Aziz'Inanga, qui contribua à son insu à une cristallisation de la gabonité autour du bwiti, ce phénomène génère parfois des discordances avec les orientations religieuses que prennent les carrières des artistes, reflétant des contradictions qui traversent la société gabonaise dans son ensemble. Celles-ci se sont dressées au fil du processus qui, au travers de plusieurs décennies, a vu les sociétés initiatiques passer du registre de la diabolisation à celui de la patrimonialisation, dans le même temps que les églises pentecôtistes gagnaient en influence et en visibilité dans l'espace public [Mebiame-Zomo, 2011]. En retraçant le parcours d'un troisième artiste, je propose d'examiner plus en détail les mouvements patrimoniaux auxquels s'associaient (consciemment ou non) les musiciens dans leurs usages des domaines initiatiques, et d'accéder à une étape supplémentaire de compréhension du processus de passage à l'art (et au patrimoine) du bwiti. 


\section{Mobilités du religieux : décontextualisation et recontextualisation des traditions initiatiques chez Vyckos Ekondo}

\section{Le bwiti et les sociétés initiatiques au Gabon : une patrimonialisation fragmentaire et ambiguë}

Condamnés durant la phase coloniale, le bwiti et les sociétés initiatiques locales sont progressivement devenus, à partir des indépendances et des années 1970, des composantes d'un patrimoine national à exposer, en tant que traits d'exception locale ayant résisté à la colonisation, et susceptibles de concourir au rayonnement des cultures gabonaises de par le monde. Ce changement de statut est survenu par l'opération croisée de différents agents, institutionnels ou non institutionnels, et par des dialogues entre ces acteurs.

Durant la période coloniale, le bwiti et d'autres sociétés initiatiques connaissent un phénomène de vivification et d'effervescence remarquables, en vue de répondre à la déstructuration violente de la colonisation [Fernandez, 1982; Gray, 2002], et au réveil pentecôtiste évangélique des années 1930 [Mary, 1999]. Né dans les ethnies mitsogho et apindji du sud-centre du Gabon, le bwiti s'est diffusé largement et rapidement dans une majorité des ethnies méridionales et occidentales, avant de se propager dans l'ethnie fang du nord du Gabon, où le rite a rencontré ses modifications les plus notoires, de par les syncrétismes avec la chrétienté [Mary, 1983a]. « Idéologie des indépendances » [Mary, 1983a, p. 343], et en pleine effervescence à partir des années 1940, le bwiti fang représente un important soutien pour l'accès à la présidence de Léon $\mathrm{M}^{\prime} \mathrm{Ba}$, présenté par les prophètes comme le messie venu libérer le peuple noir [Ibid.]. Pourtant, loin de répondre aux espoirs de reconnaissance et d'institutionnalisation, ce dernier continue durant son mandat à reléguer le bwiti au rang de « religion des villages et des quartiers », au profit de l'Église catholique.

Sous Omar Bongo, cette relégation s'apparente de plus en plus à une forme de répression, par différentes actions de contrôle, de surveillance ou d'interdiction des rituels par l'administration. Ces mesures répondent officiellement à la politique de «développement » et à « l'idéologie du travail » énoncées sous le régime de rénovation, ainsi qu'à la répression plus générale des manifestations collectives, qui sont de potentiels foyers d'opposition [Mary, 1983a, p. 348]. Omar Bongo exerce un contrôle ferme de l'influence du bwiti, ménageant de la sorte les intérêts du clergé catholique, quoiqu'il soit aussi connu pour être un haut initié du ndjobisociété de pouvoir de l'Est gabonais [Soiron, 2009].

Le contrôle exercé sur le bwiti témoigne plus largement de la représentation ambivalente de la «tradition» à l'époque. En 1974, durant le premier festival culturel gabonais, le président de l'Assemblée nationale (Georges Damas-Aleka), et d'autres invités soulignent par exemple « la nécessité de filtrer [les] traditions, pour ne conserver que les meilleures », et l'importance d'éliminer l'excès de ngozés (veillées de bwiti fang) «cause de déchéance collective et [...] de dégénérescence de la tribu» [Damas-Aleka, 1974, p. 52]. Bien que reconnus comme 
traditions, le bwiti et d'autres sociétés initiatiques ne sont donc pas considérés comme dignes de faire patrimoine dans la nation moderne.

À l'opposé de cette politique ambiguë de contrôle des religions traditionnelles, d'autres agents négocient avec l'État pour parvenir à une reconnaissance progressive de la dimension «traditionnelle» et «patrimoniale» du bwiti. Le Musée national des arts et traditions apporte une impulsion importante à cette entreprise, en corrélation avec les enquêtes des ethnologues qui y exercent : né d'une convention entre l'Orstom et l'État gabonais en 1960, en vue de recueillir les expressions traditionnelles du Gabon, ce musée est constitué à partir des collections d'ethnologues et d'ethnomusicologues. Recréant en son sein les milieux d'origine des pratiques observées, il abrite par exemple la reconstitution d'un «temple de la confrérie du Bwiti des Mitsogho » [Perrois, 1969]. Dans ce sillon, d'autres ethnologues publièrent des monographies à propos des rites initiatiques [Fernandez, 1982 ; Mary, 1983b ; Swiderski, 1990 ; Bureau, 1996], concourant à leur offrir une place centrale dans l'anthropologie gabonaise.

Plus tard, la patrimonialisation du bwiti bénéficie également pour partie de l'impulsion d'universitaires gabonais, qui organisent en 2000 à l'université Omar Bongo de Libreville un séminaire intitulé «Bwiti du Gabon » [Bonhomme, 2007]. Depuis une période plus récente, des Occidentaux s'investissent par des écrits et des productions audiovisuelles (à la télévision et au cinéma) dans la mission de faire connaître les bienfaits potentiels du bwiti et de l'iboga, pour le Gabon, l'Afrique et le monde [Chabloz, 2014]. Quelques initiateurs et personnalités bwitistes se produisent aujourd'hui occasionnellement à la télévision pour appeler à la protection de ce rite. En 2000, l'État gabonais a finalement décrété que l'iboga (plante hallucinogène employée dans le rite) représentait un «patrimoine national » et une «réserve stratégique », mais sans pour autant faire du bwiti lui-même un bien culturel à sauvegarder.

Influencés par ce contexte, par leurs mobilités et par leurs trajectoires personnelles, les musiciens se sont eux aussi impliqués dans cette « arène patrimoniale » [Givre, 2012] formée autour du changement de statut des rites initiatiques. Alors que Pierre-Claver Akendengué et Aziz'Inanga puisaient de façon secondaire dans ces rites, sans avoir traversé l'initiation, le chanteur Vyckos Ekondo y accorda une place centrale, en affirmant son statut d'initié et en bouleversant certains interdits initiatiques.

\section{Spectacularisation du religieux initiatique et controverses patrimoniales}

Né en 1953 à Mimongo (sud du Gabon), Vyckos Ekondo est élevé dans une famille de l'ethnie mitsogho, à la fois chrétienne et versée dans les rites traditionnels ${ }^{11}$. Comme beaucoup de jeunes ruraux, il baigne dans cet environnement

11. L'Agence de coopération culturelle et technique (ACCT) est l'ancêtre de l'Organisation internationale de la Francophonie (OIF). 
initiatique, sans pour autant s'initier. Il part à l'âge de onze ans à Libreville pour y être scolarisé au collège Felix Éboué, où il découvre le solfège, la musique classique et le piano. Tout en continuant ses études, il commence à animer des émissions de variétés à la télévision nationale (la RTG) et réalise des programmes sur les folklores gabonais, s'instruisant ainsi sur les danses et cérémonies pratiquées dans les neuf provinces de son pays. Il se fait progressivement connaître dans le monde de la chanson pour son style mêlant soul, funk et disco, et ses shows accompagnés de ses «Vyckossettes ».

Les circulations internationales de Vyckos Ekondo vont être un premier levier du projet de gabonisation et d'africanisation de sa musique. En 1975, il approfondit sa formation en communication à l'Université du Québec. Lorsqu'il présente à ses enseignants des interprétations de morceaux d'artistes célèbres, ainsi qu'un conte traditionnel mitsogho, ceux-ci l'incitent à privilégier « la musique de chez lui ». Il prend dès lors conscience de l'importance de mettre en avant des éléments de son «folklore» pour s'insérer dans le marché du spectacle international.

De retour au Gabon, Vyckos poursuit son travail à la télévision gabonaise, anime des émissions de variétés populaires, et évolue au sein de réseaux médiatiques sous la houlette du parti. À partir des années 1980, il commence à imposer un nouveau style sur les scènes musicales populaires, en prônant la diffusion des sonorités et des danses traditionnelles. Interrogé à propos de ce déplacement vers un genre musical «tradi-moderne », Vyckos Ekondo explique qu'il s'engouffre alors dans la brèche ouverte par le président Bongo quelques années précédemment, lorsqu'il proclamait ainsi : " mon soutien est acquis à tous ceux qui s'attachent à la préservation de l'originalité et de la personnalité gabonaise » [Bongo cité par Owono Nguéma, 1974, p. 5]. Au contraire d'un « retour aux sources » potentiellement subversif, Vyckos Ekondo reproduit soigneusement les injonctions à l'unité nationale du système politique en créant un spectacle de la mosaïque ethnique, et il suit les évolutions des politiques culturelles des années 1980, qui manifestent une ouverture aux «traditions » et au «patrimoine $»^{12}$.

D'abord avec des danseurs du ballet national, puis avec sa troupe Tandima, Vyckos Ekondo crée les premiers vidéoclips et chorégraphies reprenant des cérémonies d'initiation, des danses de réjouissance, de circoncision et de sortie de masque des neuf provinces. Il crée des accessoires scéniques et des costumes aux couleurs des rites initiatiques, il reprend les maquillages au kaolin et les tenues de pagne ou de raphia qu'on arbore lors des veillées, et il élabore des chorégraphies agençant les mouvements des initiés. Il utilise aussi dans ses enregistrements les sonorités des instruments caractéristiques du bwiti-les trompes en corne d'antilope (ghétsika mbudi), des harpes ngombi et des arcs musicaux mongongo.

12. Cette évolution des politiques culturelles se manifeste par la participation à la conférence de 1'Unesco sur les politiques culturelles tenue à Mexico en 1982, et par la création en 1983 du CICIBA (Centre international des civilisations bantu). Ce centre connut un certain rayonnement dans les années 1980, mais fut rapidement laissé à l'abandon. 
Certains de ses vidéoclips font alors scandale dans les milieux d'initiés, et déclenchent les foudres des aînés initiatiques, en raison de la profanation des secrets ou les usages de parures réservées à de hauts gradés qu'ils exposent. Dans le clip du morceau «Bovenga Ngoye » (Safari Ambiance, 1990), Vyckos met en scène des danses de masques et des costumes réservés aux initiés, ainsi que des symboles cosmogoniques, tel que l'œuf originel. Certains de ses danseurs seront contraints, pour expier leur infraction aux interdits initiatiques, de payer symboliquement des « amendes » auprès des instances où ils étaient initiés.

Au fil de sa carrière, Vyckos traverse lui aussi les voies de l'initiation, afin de maîtriser les dimensions sacrées du bagage dont il s'empare et d'acquérir la légitimité requise pour assumer le rôle d'ambassadeur de cette tradition initiatique :

«Quand j'ai commencé la musique, je faisais la musique comme tout le monde. Dès que j'ai commencé à exploiter ce qui était du bwete, mon père m'avait exigé que je m'initie. [...] Je me suis fait d'abord initier. Au retour, j'ai fait enregistrer mon premier disque. Donc j'ai fait la part des choses, je savais ce qui était sacré et ce qui était à la portée du public. C'était pour moi aussi de montrer que nos sociétés secrètes ne sont pas là pour faire du fétichisme, mais montrer aussi la contribution du bwete dans le rayonnement spirituel et culturel du Gabon. » (Vyckos Ekondo, mars 2012, Libreville)

Comme le laisse entendre Vyckos, le processus de décontextualisation et de recontextualisation en œuvre dans ses mises en scène de symboles religieux s'appuie sur l'existence, au sein du bwiti, d'une distinction entre une partie secrète et une partie publique du rite. En effet, les veillées de bwiti (comme d'autres rites initiatiques) comportent une dimension publique et sont accessibles aux profanes, qui prennent place dans une partie réservée du temple. D'après Vyckos, ses spectacles ne transgresseraient pas cette frontière instituée. Cependant, en franchissant une étape inédite dans l'utilisation des symboles religieux sur scène et dans ses clips, et en montrant des objets jusqu'alors dissimulés, Vyckos entraîna des controverses auprès d'initiés et de pairs musiciens ou danseurs. Outre la dénonciation de la divulgation du secret initiatique, des initiés récusaient son autorité sur les chants et phrases chorégraphiques repris du répertoire traditionnel. D'autres rejetaient enfin le choix de spectaculariser des traditions encore bien vivaces chez une partie de la population, et qui ne nécessitaient donc ni «revalorisation» ni modification à des fins de survivance.

Le processus de décontextualisation et de mise en scène du bwiti engagé par Vyckos Ekondo déclenchait donc des résistances remettant en question la légitimité du chanteur et critiquant la dissolution des significations symboliques au profit de la marchandisation de pratiques rituelles. Autour de «l'artification» [Heinich, Shapiro, 2012] d'une pratique initiatique et des symboles qui en sont issus, émergent ainsi des conflits. Ces mêmes conflits s'observent lorsque des universitaires organisent des conférences sur le bwiti aux côtés d'initiés [Bonhomme, 2007], qui se refusent à la publicisation de leurs savoirs, récusent la nécessité de valorisation d'une pratique bien vivace, et résistent in fine, à sa patrimonialisation. 


\section{Des musiciens dans « l'arène patrimoniale »: de l'invention de la tradition au façonnage du patrimoine}

À des époques et selon des degrés variés, les trois artistes ici décrits ont chacun contribué à une opération de façonnage et de production d'un patrimoine autour d'éléments religieux initiatiques, en lien avec les influences des ethnologues, des politiques culturelles et des marchés musicaux. Alors que les institutions d'archives et les musées restent rares ou inaccessibles au commun des Gabonais, ces musiciens diffusés auprès d'une large partie des foyers gabonais ont été investis du rôle d'ambassadeurs culturels et de passeurs de patrimoines. Certains, comme Vyckos Ekondo sont aujourd'hui sollicités pour donner des enseignements dans le cadre de formations universitaires sur les métiers du patrimoine, d'autres comme Pierre-Claver Akendengué ont exercé les fonctions de conseillers à la présidence ; tous sont invités fréquemment pour des festivités, et leurs musiques sont reprises (parfois au travers de la méthode du sampling ou de l'échantillonnage sonore) par de jeunes artistes désireux de s'affilier à des personnalités porteuses d'un héritage commun.

Les carrières de ces trois musiciens ont pourtant connu des destins différenciés : celle d'Aziz'Inanga a rapidement décliné après sa séparation d'avec son conjoint et son départ en France ; ses relations ambivalentes avec le pouvoir, nées entre autres de son appartenance à un groupe ethnique lié à l'opposition et sa dépendance à l'égard d'un mentor masculin ont sans nul doute été à l'origine de ce déclin, la positionnant dans une posture de dépendance et de vulnérabilité. Comme elle, Pierre-Claver Akendengué dut négocier avec l'instrumentalisation des musiciens, et avec les envers de la "géopolitique » du système Bongo qui surveille étroitement les activités de certains groupes ethniques ; pourtant ses soutiens solides dans des réseaux à l'intérieur et au-delà du Gabon l'autonomisèrent rapidement de ce régime de contrôle. Sa reconnaissance dans les marchés de la world-music lui permit d'acquérir ce statut comme passeur et ambassadeur des «traditions » gabonaises. Pour sa part, Vyckos Ekondo est parvenu à construire un style en accord avec les formats imposés par les projets nationalistes de l'État gabonais, opérant à la fois au sein des institutions culturelles et en dehors.

En retraçant leurs parcours, cet article a mis en évidence l'importance des mobilités dans les vies personnelles, les parcours religieux et les influences artistiques de ces musiciens, et il a permis d'identifier plusieurs types de circulations créatrices ayant influencé leur manière de penser et de faire le patrimoine. Au travers de leurs mobilités sur les routes du panafricanisme, les marchés de la world-music ou les chemins nostalgiques du village et de ses «traditions » fantasmées, ces chanteurs contribuèrent à investir certains objets ou éléments de tradition d'un pouvoir iconique, ou encore du rôle de "sémiophore» dans la fabrique et la transmission d'une histoire culturelle [Pomian, 2010].

En abordant la patrimonialisation du bwiti par l'entrée musicale, cet article confirme combien l'étude des pratiques musicales et des trajectoires d'artistes permet d'accéder à la compréhension des mécanismes de négociations avec le 
pouvoir induit par la fabrique du patrimoine : en considérant ces musiques en lien avec leurs contextes politiques, leur dimension multiscalaire et leurs enjeux marchands, on accède à une description dense des tensions, des ambiguïtés et des négociations soulevées par la patrimonialisation. Cette approche révèle les doubles visages revêtus par la «tradition» dans le projet de construction d'un État gabonais moderne, et la manière dont ce rapport ambigu au religieux « traditionnel » s'est répercuté dans le processus patrimonial. «Tradition» et «patrimoine » se répondent à nouveau ici, employés pour façonner des héritages du passé en fonction d'intérêts présents.

Finalement, l'étude de ces musiciens révèle comment la patrimonialisation et les nationalismes ne s'élaborent ni en totale contradiction ni en adhésion ni en décalage avec le projet étatique, mais en négociant avec son contrôle et ses cadres idéologiques. On s'aperçoit alors que là où les tensions et les intérêts divergents entre les acteurs de « l'arène patrimoniale » [Givre 2012] ont jusqu'à présent fait échouer la reconnaissance officielle du bwiti comme religion nationale, les musiciens ont contribué à créer une nouvelle représentation de ces pratiques et à les instituer, au moins dans l'opinion populaire, en un «patrimoine » national, caractérisant le Gabon sur le plan local et international.

\section{Bibliographie}

Adi H., SHerwood M. [2003], Pan-African history : political figures from Africa and the diaspora since 1787, London, Routledge.

AKendengue P.C. [1986], Religion et éducation traditionnelles en pays nkomi au XIXe siècle, thèse de doctorat en sociologie, Paris, Université Paris 5.

Akendengue P.C., Thiéfaine J. [1996], « Pierre Akendengué. Pionnier, conteur et humaniste », Chorus. Les cahiers de la chanson, $\mathrm{n}^{\circ} 17, \mathrm{p} .130-134$.

Akendengue P.C., ThiÉFAIne J. [2008], «"Il y a chez moi une troisième dimension liée à la quête de la spiritualité". Entretien de Gérald Arnaud avec Pierre Claver Akendengue », Africultures : http://africultures.com/il-y-a-chez-moi-une-troisieme-dimension-liee-a-la-quete-de-la-spiritualite7389/ (page consultée le 7 février 2017).

Amselle J.-L. [2001], Branchements. Anthropologie de l'universalité des cultures, Paris, Flammarion.

Aterianus-Owanga A. [à paraître], « Cultural animation groups », in FeldMAN H. (dir.) Encyclopedia of popular music of the world, african genres, New York (N. Y.), Bloomsbury Publishing.

Aterianus-Owanga A. [2016], «"Tu t'en es pris à la mauvaise go !" Transgresser les normes de genre sur les scènes rap du Gabon », Ethnologie française, $\mathrm{n}^{\circ}$ 46, p. 45-58.

Aterianus-Owanga A. [2015], «Femme-panthère, homme caméléon. Animalisation du soi et rhétorique de l'authenticité chez les musiciens du Gabon», Religiologiques, $\mathrm{n}^{\circ} 32$, p. 299-326.

Bernault F. [1996], Démocraties ambiguës en Afrique centrale, Paris, Karthala. 
Bondaz J., Graezer Bideau F., Isnart C., Leblon A. [2014], Les Vocabulaires locaux du "patrimoine ». Traductions, négociations et transformations, Zurich, Berlin, Lit Verlag.

Bonhomme J. [2006], Le Miroir et le Crâne. Le parcours initiatique du bwete misoko (Gabon), Paris, CNRS, Maison des sciences de l'homme.

BonHomme J. [2007], « Anthropologue et/ou initié. L'anthropologie gabonaise à l'épreuve du bwiti », Journal des anthropologues, $\mathrm{n}^{\circ}$ 110-111, p. 207-226.

Boukari-Yabara A. [2014], Africa Unite! Une histoire du panafricanisme, Paris, La Découverte.

Buscatto M. [2007], Femmes du jazz. Musicalités, féminités, marginalisations, Paris, CNRS.

CAmpos L. [2011], «Sauvegarder une pratique musicale? Une ethnographie du samba de roda à la World Music Expo », Cahiers d'ethnomusicologie, no 24, p. 143-155.

Chabloz N. [2014], Peaux blanches, racines noires. Le tourisme chamanique de l'iboga au Gabon, Paris, Louvain-la-Neuve, L'Harmattan, Academia.

Charles-Dominique L. [2013], «La Patrimonialisation des formes musicales et artistiques: anthropologie d'une notion problématique », Ethnologies, vol. 35, n ${ }^{\circ}$ 1, p. 75-101.

CiARCIA G. (dir.) [2011], Ethnologues et passeurs de mémoires, Paris/Montpellier, Karthala/ Maison des sciences de l'homme de Montpellier.

Damas-Aleka G. [1974], « Tradition et modernité », in République Gabonaise, Gabon, Premier festival culturel, Culture et développement, actes du colloque, Libreville, 16-26 mars 1974, ministère de la Culture et des Arts, p. 49-55.

Desroches M., Dauphin C., Pichette M.-H., Smith G.E. (dir.) [2011], Territoires musicaux mis en scène, Montréal, Les Presses de l'Université de Montréal.

Dozon J.-P. [2015], Afriques en présence. Du monde Atlantique à la globalisation, Paris, Maison des sciences de l'homme.

FABRE D. [1994], «Ethnologie et patrimoine en Europe», Terrain, no 22, p. 145-50.

FELD S. [2004], «Une si douce berceuse pour la "World Music" ", L'Homme, no 171-172, p. $389-408$.

FERNANDEZ J. [1982], Bwiti : an ethnography of the religious imagination in Africa, Princeton (N. J.), Princeton University Press.

Gilroy P. [2010], L'Atlantique noir. Modernité et double conscience, Paris, Éditions Amsterdam.

GIvRE O. [2012], « Savoirs et pouvoirs, stratégies et tactiques dans "l'arène patrimoniale" du nestinarstvo (Bulgarie) », Civilisations, n 61, p. 103-120.

Givre O., Regnault M. (dir.) [2015], Patrimonialisations croisées. Jeux d'échelles et enjeux de développement, Lyon, Presses universitaires de Lyon.

Gray C.J. [2002], Colonial rule and crisis in Equatorial Africa: Southern Gabon, ca. 1850-1940, Rochester (N. Y.), University of Rochester Press.

Heinich N., Shapiro R. (dir.) [2012], De l'artification. Enquêtes sur le passage à l'art, Paris, EHESS.

Hervieu-Leger D. [1993], La Religion pour mémoire, Paris, Éditions du Cerf.

HugGan G. [2001], The postcolonial exotic : marketing the margins, London, Routledge.

ISNART C. [2012], «Les patrimonialisations ordinaires. Essai d'images ethnographiées », ethnographiques.org, $\mathrm{n}^{\circ} 24$ : www.ethnographiques.org/2012/ (page consultée le 13 avril 2016). 
LE GofF J. (dir.) [1998], Patrimoine et passions identitaires, Actes des entretiens du patrimoine, Paris, Fayard, Édition du Patrimoine.

Le Menestrel S. (dir.) [2012], Des vies en musique. Parcours d'artistes, mobilités, transformations, Paris, Hermann.

Mapangou L.B., Barbier-Decrozes M. [1985], Mémorial du Gabon, 1960-1985, tome 3, 1975-1979, Le Faste et la Rigueur, Genève/Monaco, SYNER.

MARY A. [1999], Le Défi du syncrétisme. Le travail symbolique de la religion d'Eboga (Gabon), Paris, EHESS.

MARY A. [1983a], «L'alternative de la vision et de la possession dans les sociétés religieuses et thérapeutiques du Gabon », Cahiers d'études africaines, vol. 23, nº 91, p. 281-310.

MARY A. [1983b], La Naissance à l'envers : essai sur le rituel du Bwiti-Fang au Gabon, Paris, L'Harmattan.

MARY A. [2005], «Le Bwiti à l'heure du village global», Rupture-solidarité, nº 6, p. 83-103.

M'BокоLо E. [2009], Médiations africaines. Omar Bongo et les défis diplomatiques d'un continent, Paris, Hachette.

MbONDOBARI S. [2004], « Regard critique sur l'Afrique coloniale et postcoloniale à partir de la chanson francophone d'Afrique. Le cas de l'œuvre musicale de Pierre-Claver Akendengué », in Mukala K N., Malonga A.N. (dir.), Itinéraires et convergences des musiques traditionnelles et modernes d'Afrique, Paris, FESPAM, L'Harmattan, p. 223-240.

Metegue N'Nah N. [2006], Histoire du Gabon: des origines à l'aube du XXI" siècle, Paris, L'Harmattan.

Mouele P.N. [2009], Spectacularisation du corps féminin et construction de l'hégémonie politique au Gabon Postcolonial, mémoire de maitrise en sociologie, Libreville, Université Omar Bongo.

Mve Bekale M. [2000], Pierre-Claver Zeng et l'art poétique fang. Esquisse d'une herméneutique, Paris, L'Harmattan.

Ndiouga Benga A. [2008], « Mise en scène de la culture et espace public au Sénégal, 1960-2000», $12^{\mathrm{e}}$ assemblée du CODESRIA, Administrer l'espace public africain, Décembre 2008, Yaoundé.

Nguema Minko E. [2010], Gabon : l'unité nationale ou la rancune comme mode de gouvernance, Paris, L'Harmattan.

Obiang J.-F. [2007], France-Gabon : pratiques clientélaires et logiques d'État dans les relations franco-africaines, Paris, Karthala.

OLIVIER E. [2011], «Logique patrimoniale de la musique et globalisation musulmane à Djenné (Mali) », in DesRoches M. (dir.), Mise en scène des territoires musicaux : tourisme, patrimoine et performance, Montréal, Presses de l'Université de Montréal, p. 216-233.

Ondo P., Ovono ÉBE M. [2014], Regards sur l'œeuvre artistique d'un poète-philosophe fang : Pierre Claver Zeng, Libreville, ODEM.

Owono-Nguema F. [1974], «Introduction », in République Gabonaise, Gabon, Premier festival culturel national, colloque sur le thème : cultures et développements, actes du colloque, Libreville, ministère de la Culture et des Arts, p. 5-8.

Perrois L. [1969], Gabon: Culture et techniques. Musée des arts et traditions, Libreville, Orstom : http://horizon.documentation.ird.fr/exl-doc/pleins_textes/pleins_textes_5/b_fdi_ 04-05/05035.pdf (page consultée le 14 avril 2016). 
Perrois L. [1999], «La formation d'un "patrimoine" du Sud : le musée des Arts et Traditions du Gabon », Ethnologie française, vol. 29, n 3, p. 345-354.

Pomian K. [2010], «Patrimoine et identité nationale », Le Débat, nº 159, p. 45-56.

Pourrouquet A. [2015], «La production du patrimoine musical malien», in Givre O., RéGnault M. (dir.), Patrimonialisations croisées. Jeux d'échelles et enjeux de développement, Lyon, Presses universitaires de Lyon.

Retondah G.S.T. [2012], Ezélé ! Pierre Akendengué, un cri de liberté, Libreville, Éditions Raponda-Walker.

Rich J. [2012], «Gabonese men for french decency : the rise and fall of the gabonese chapter of the Ligue Des Droits de l'Homme, 1916-1939», French colonial history, vol. 13, n 1, p. 23-53.

RoDA J. [2011], « Des Judéo-Espagnols à la machine unesquienne. Enjeux et défis de la patrimonialisation musicale », Cahiers d'ethnomusicologie, $\mathrm{n}^{\circ}$ 24, p. 123-141.

SAlLÉe P. [1985], L'Arc et la Harpe. Contribution à l'histoire de la musique du Gabon, Paris, Thèse de docteur de troisième cycle, Université Paris X.

SAMSON G., SANDRONI C. [2013], «The recognition of Brazilian samba de roda and reunion maloya as intangible cultural heritage of humanity », Vibrant : Virtual Brazilian. Anthropology, vol. 10, $\mathrm{n}^{\circ} 1$ : www.vibrant.org.br/issues/v10n1/guillaume-samson-carlos-sandronithe-recognition-of-brazilian-samba-de-roda-and-reunion-maloya-as-intangible-culturalheritage-of-humanity/ (page consultée le 14 avril 2016).

SoIron M. [2009], La Longévité Politique : ou les fondements symboliques du pouvoir politique au Gabon, Lille, thèse de doctorat en sociologie, Université Lille 1.

SwidersKi S. [1990], La Religion Bouiti, vol. 1, Histoire (1840-1948), Ottawa, Legas.

Tindy-Poaty J.J. [2008], Pierre Claver Akendengué ou l'épreuve du miroir, Paris, l'Harmattan.

White B.W. [2006], «L'incroyable machine d'authenticité : l'animation politique et l'usage public de la culture dans le Zaïre de Mobutu», Anthropologie et sociétés, vol. 30, $\mathrm{n}^{\circ} 2$ : http://id.erudit.org/iderudit/014113ar (page consultée le 14 avril 2016).

White B.W. (dir.) [2012], Music and globalization, critical encounters, Bloomington (Ind.), Indiana University Press.

\section{Discographie}

Akendengue P.C. [1976], Afrika Obota, Saravah.

AzIz'Inanga [1979], A kuku. Nostalgie des pirogues à voile, Ivanga Productions. 